\title{
Optimal fluoroscopic viewing angles for stenting of the coronary aorto-ostial lesions
}

\author{
Radosław Targoński ${ }^{1}$, Jarosław Meyer-Szary ${ }^{2}$, Bartosz Baścik $^{3}$, Edyta Szurowska ${ }^{3}$, \\ Aleksandra Gąsecka ${ }^{4}$, Dariusz Jagielak ${ }^{1}$, Miłosz J. Jaguszewski ${ }^{5}$ \\ ${ }^{1}$ Department of Cardiac and Vascular Surgery, Medical University of Gdansk, Poland \\ ${ }^{2}$ Department of Pediatric Cardiology and Congenital Heart Diseases, Medical University of Gdansk, Poland \\ ${ }^{3}$ Department of Radiology, Medical University of Gdansk, Poland \\ ${ }^{4}$ First Chair and Department of Cardiology, Medical University of Warsaw, Poland \\ ${ }^{5}$ First Department of Cardiology, Medical University of Gdansk, Poland
}

\section{This paper was guest edited by Prof. Zenon Huczek}

\begin{abstract}
Background: Long-term results after stenting aorto-coronary ostial lesions (AOL) are worse than those achieved in non-ostial locations. AOL interventions still pose a substantial challenge for interventional cardiologists. The aim of the study was to determine the optimal fluoroscopic viewing angles of the left and right coronary ostia, based on multislice computed tomography (MSCT) data.

Methods: Cardiac MSCT exams of 30 patients with clinical suspicion of coronary artery disease were analyzed. En face angles of both coronary ostia, as well as their optimal projection curves, were determined by 2 independent observers in a standard Dicom viewer, without any additional, specialized software add-ons, using a systematic, step-by-step approach. Spatial relations between the ostial plane and the aorta were also assessed.

Results: The average en face angle of the left coronary ostium was RAO 23, CAU 45'; for the right coronary ostium $R A O 18^{\circ}, C R A 5^{\circ}$. The mean inter-observer differences for the en face angles of the left and right coronary arteries were $5^{\circ}$ and $7^{\circ}$, respectively.

Conclusions: Multislice computed tomography data provide precise spatial information on the orientation of the coronary ostia and their relation to the aortic root. Their utilization for determining the patient-specific viewing angle may substantially facilitate percutaneous coronary interventions in AOL. (Cardiol J 2021; 28, 6: 831-841)
\end{abstract}

Key words: aorto-ostial lesions, coronary intervention, optimal projection curves, multislice computed tomography, cardiovascular imaging

\section{Introduction}

Despite constant advances in technology, interventions of the coronary ostia still pose a substantial challenge for interventional cardiologists. Long-term results after stenting of the aorto-coronary ostial lesions (AOL) are worse than those achieved in non-ostial locations [1,2]. The gap results partly from differences in the plaque composition, which is more rigid, calcified, and bulky in the case of AOL [3, 4]. A lot of target lesion failures are due to technical problems during

Address for correspondence: Radosław Targoński, MD, PhD, Department of Cardiac and Vascular Surgery, Medical University of Gdansk, ul. Sklodowskiej-Curie 3a, 80-210 Gdańsk, Poland, tel: +48 607921 300, fax: +4858 5844210 , e-mail: rtargonski@gmail.com

Received: 15.03.2021 Accepted: 16.07.2021 Early publication date: 2.08.2021

This article is available in open access under Creative Common Attribution-Non-Commercial-No Derivatives 4.0 International (CC BY-NC-ND 4.0) license, allowing to download articles and share them with others as long as they credit the authors and the publisher, but without permission to change them in any way or use them commercially. 
the procedure, resulting in overly deep stent implantation and incomplete coverage of the treated lesion [5]. On the other hand, excessive protrusion of the stent into the aorta also has negative consequences because it can hinder re-engagement of the vessel with the catheter or seriously complicate subsequent interventions on the aortic valve [6,7]. Over the last several years, multi-sliced computed tomography (MSCT) has emerged as a useful tool in the assessment of coronary artery disease (CAD) [8-10]. This diagnostic modality offers a unique view into the spatial relationships of different cardiac structures and has been proved to be critical in procedure planning and device selection in structural heart interventions [11-14]. However, three-dimensional (3D) data provided by MSCT are still rarely used to facilitate percutaneous coronary interventions (PCI) $[15,16]$.

The aim of this study was to determine the optimal fluoroscopic viewing angles for AOL stenting with the use of MSCT examinations of patients with CAD. Patient-specific optimization of the viewing angle can potentially decrease/eliminate parallax error [12] and improve the precision of stent implantation in the coronary ostium. We propose a simple, step-by-step approach to find individually optimized projections with the use of patient MSCT data.

\section{Methods}

\section{Setting and image acquisition}

Multislice computed tomography examinations of 30 patients with clinical suspicion of CAD were retrospectively analyzed. MSCT was performed according to the established institutional protocol, i.e. in spiral/helical acquisition mode with retrospectively electrocardiogram-gated reconstruction, with a tube voltage of $100-120 \mathrm{kV}$, and tube current adjusted for patient size. $80-110 \mathrm{~mL}$ of contrast was injected into the antecubital vein at a rate of $5 \mathrm{~mL} / \mathrm{s}$. The dataset of contrast-enhanced scans was reconstructed in the diastolic phase. Images were reconstructed with a slice thickness of $0.6 \mathrm{~mm}$ and a reconstruction interval of $0.4 \mathrm{~mm}$.

\section{Image analysis}

Multislice computed tomography exams were then analyzed with a standard DICOM viewer (Osirix Pixmeo SARL, Geneva, Switzerland). A step-by-step approach, described in detail in Figure 1, was used to calculate the en face angles of the coronary aorto-ostial orifice plane. Based on the determined en face angles, optimal projection curves (S-curve) (Fig. 2A) were then calculated in an Excel (Microsoft Corporation Redmond, WA, USA) spreadsheet using the following formula described earlier by the Nicolo Piazza group [17]:

$$
\varnothing=-\arctan \left[\frac{\cos \theta-\theta_{\text {enface }}}{\tan \emptyset_{\text {enface }}}\right]
$$

where $\emptyset$ is the cranio-caudal angle of the optimal projection curve at RAO/LAO angle $\theta$, and $\emptyset_{\text {enface }}$ and $\theta_{\text {en face }}$ are the cranio-caudal and RAO/LAO angles of the structure viewed en face, respectively (online version of the S-curve generator is available at https://smartheart.pl/plane-calculator/). The multiplanar reconstruction mode was applied again to define the range of the S-curve in which the adjacent aorta does not overlap with the coronary ostium in the orthogonal angiographic view (Figs. $2 \mathrm{~B}, 3)$. Each exam was analyzed independently by two cardiologists with experience in cardiac computed tomography (CT) interpretation.

\section{Statistical analysis}

En face angles are expressed as mean with 95\% confidence interval (CI), taking the average value from both observers. Discrepancies between the investigators were assessed by measuring the average angle between the S-curves produced by each investigator.

The institutional review board approved the study with a waiver of informed consent.

The numerical data underlying this article and the anonymized CT scan data will be shared on reasonable request to the corresponding author.

\section{Results}

Table 1 presents mean en face angles of the left and right coronary ostial planes (RAO $29^{\circ}$, CAU $50^{\circ}$; and RAO $18^{\circ}, \mathrm{CRA}^{\circ}$, respectively). The mean inter-observer differences for the en face angles of the left and right coronary arteries (LCA, RCA) were $5^{\circ}$ and $7^{\circ}$, respectively. The average range of the optimal projection curve in which the left coronary ostium does not overlap with the adjacent aorta was RAO $0^{\circ}$ to LAO $68^{\circ}$. In the case of the right coronary ostium S-curve, that range was outside of the RAO $37^{\circ}$ to LAO $48^{\circ}$ zone (Fig. 4).

Figure 5 shows individual projection curves for each patient, the range of "unobstructed" view, and en face angles. Mean optimal projection curves, mean "unobstructed" view ranges, and mean en face angles for the left and right coronary ostia with 95\% CI are presented in Figure 4. 


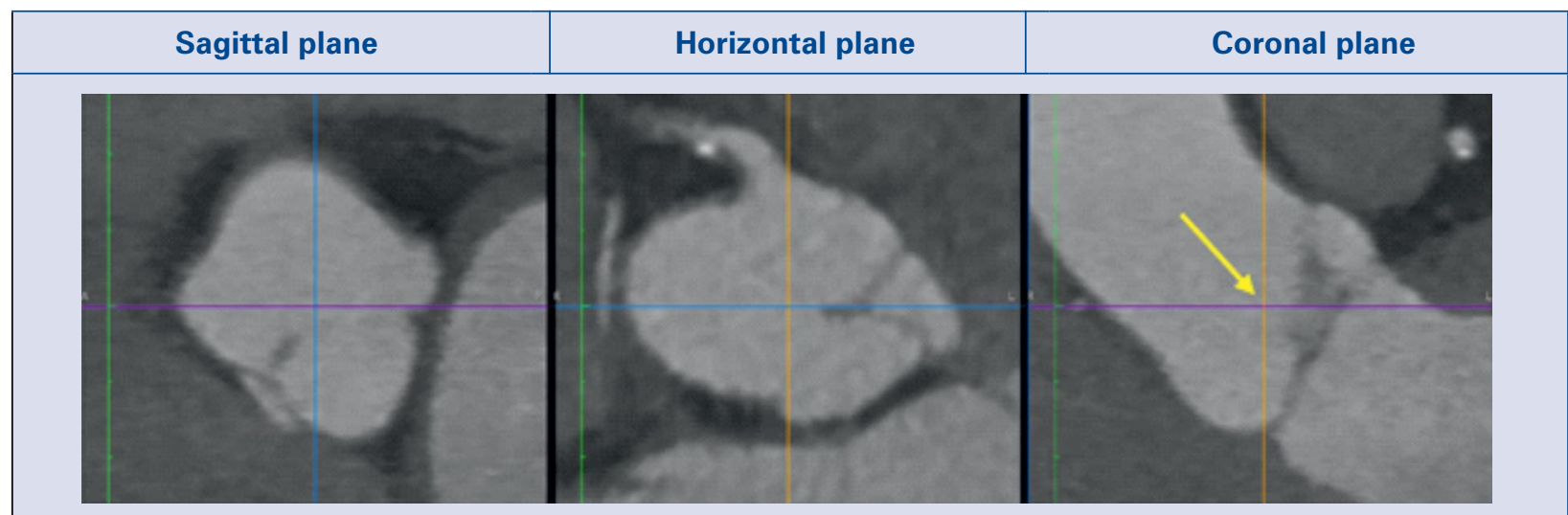

Step 1. Place the crosshair at the center of the aortic root (arrow).

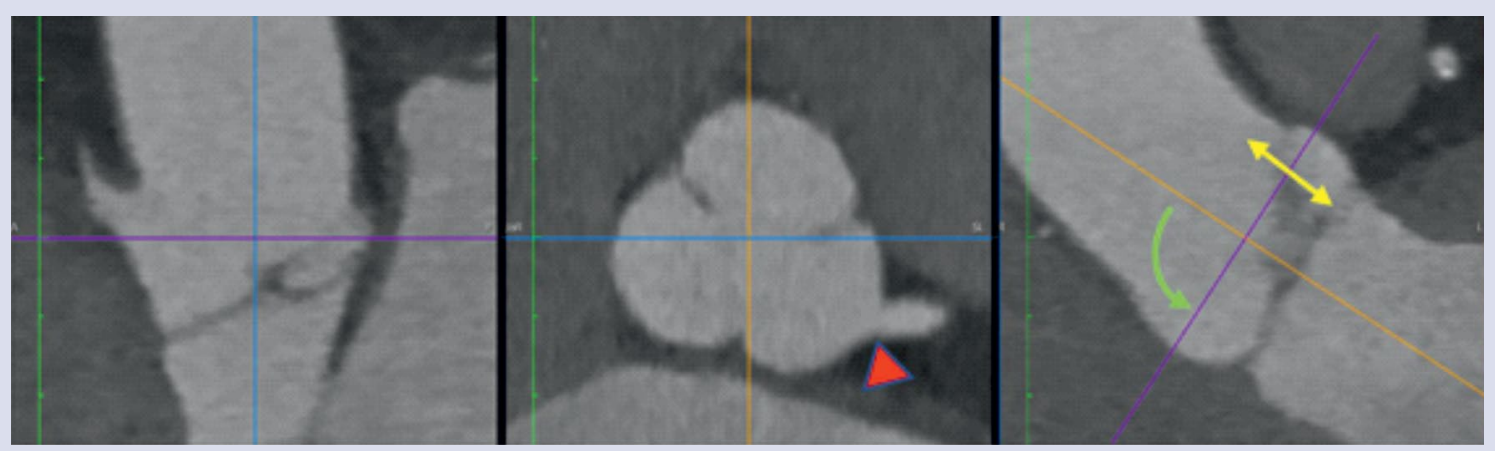

Step 2. In the coronal view window - adjust the horizontal plane (purple line) to be roughly parallel to the aortic annulus (green arrow), then adjust the horizontal plane's position (yellow arrow) to the level of the coronary ostium of interest (red arrowhead).

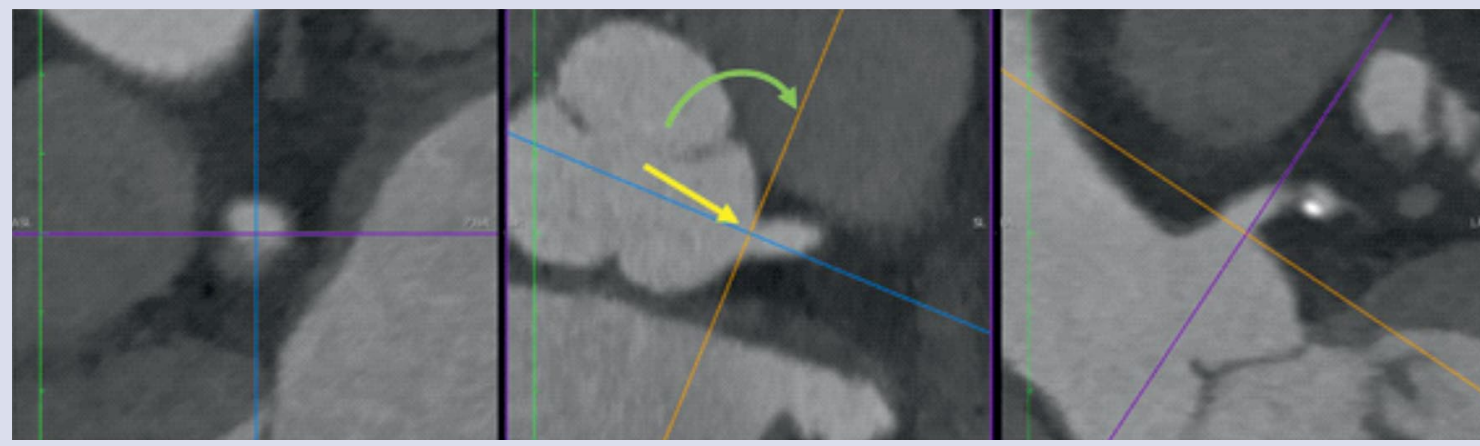

Step 3. In the horizontal view window - move the crosshairs to the center of the coronary ostium (yellow arrow), and align the sagittal plane tangentially (green arrow) to the aortic root at the level of the ostium.

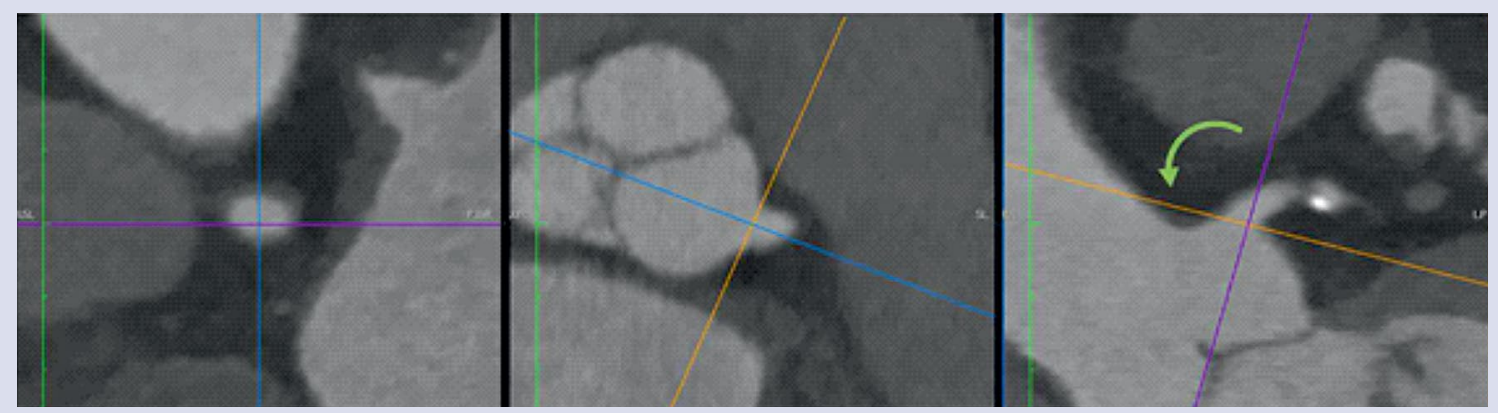

Step 4. In the frontal view window - adjust the sagittal plane tangentially to the aortic root at the level of the ostium (green arrow). 


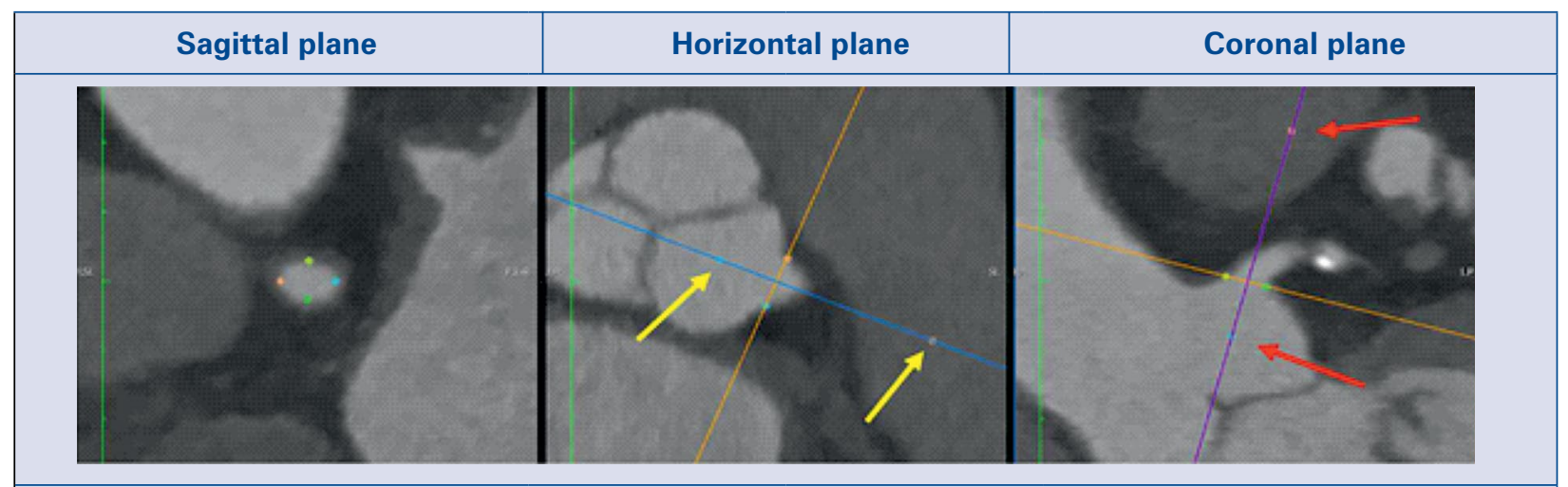

Step 5. In the frontal view window, place two region of interest (ROI) points on the horizontal plane (purple line, red arrows) - they will also appear on the frontal plane (blue line, yellow arrows) in the horizontal view window. In the sagittal plane, put ROI points at the edges of the coronary ostium.

\begin{tabular}{|c|c|}
\hline Before alignment & After alignment \\
\hline &
\end{tabular}

Step 6. Change the view to a three-dimensional maximal intensity projection (3D-MIP) and adjust the brightness and contrast settings to visualize optimally the point region of interest (ROI), then align together the two ROls marking the axis of the ostial plane (yellow arrows). After the alignment, correct the Roll to 0.0. At that point, the superior-inferior (S-I) angle will reflect the CAU/CRA angulation of the coronary ostium en face angle, and the left-right (L-R) angle will reflect the RAO/LAO angulation of the coronary ostium en face angle (RAO and CAU angles are in the negative range). Based on the determined en face angle, the optimal projection curve (S-curve) is drawn (Fig. 2A); CAU - caudal; CRA — cranial; RAO — right anterior oblique; LAO — left anterior oblique.

Figure 1. Systematic step-by-step approach for identifying the coronary aorto-ostial plane.

\section{Discussion}

The present study determined for the first time the optimal fluoroscopic viewing angles of the LCA and RCA based on MSCT data. This study is interesting from many perspectives: i) first and foremost, we propose a systematic stepby-step approach to determine individual optimal angiographic viewing angles for stenting of the coronary ostia based on patient angio-CT data; and ii) it provides a detailed description of the method, together with a graphic illustration of the concept. Our approach does not need any dedicated software and, thus, can be applied widely in the routine clinical practice.

\section{Percutaneous coronary interventions} of aorto-ostial lesions remain challenging

Aorto-ostial lesions are usually defined as coronary artery stenosis greater than $50 \%$, within $3 \mathrm{~mm}$ of its origin [18]. They constitute around $3 \%$ of all percutaneously treated lesions. The procedural success and clinical outcomes of these lesions are inferior to those of non-ostial locations. The difference results partly from the lesions' distinct plaque composition, causing them to be more bulky, non-compliant, and with a greater tendency towards elastic recoil. The lack of adventitia in the transmural segment may also play a role $[2,3,19]$. However, a suboptimal result is very often caused by the challenges of ostial intervention and imprecise stent positioning. 


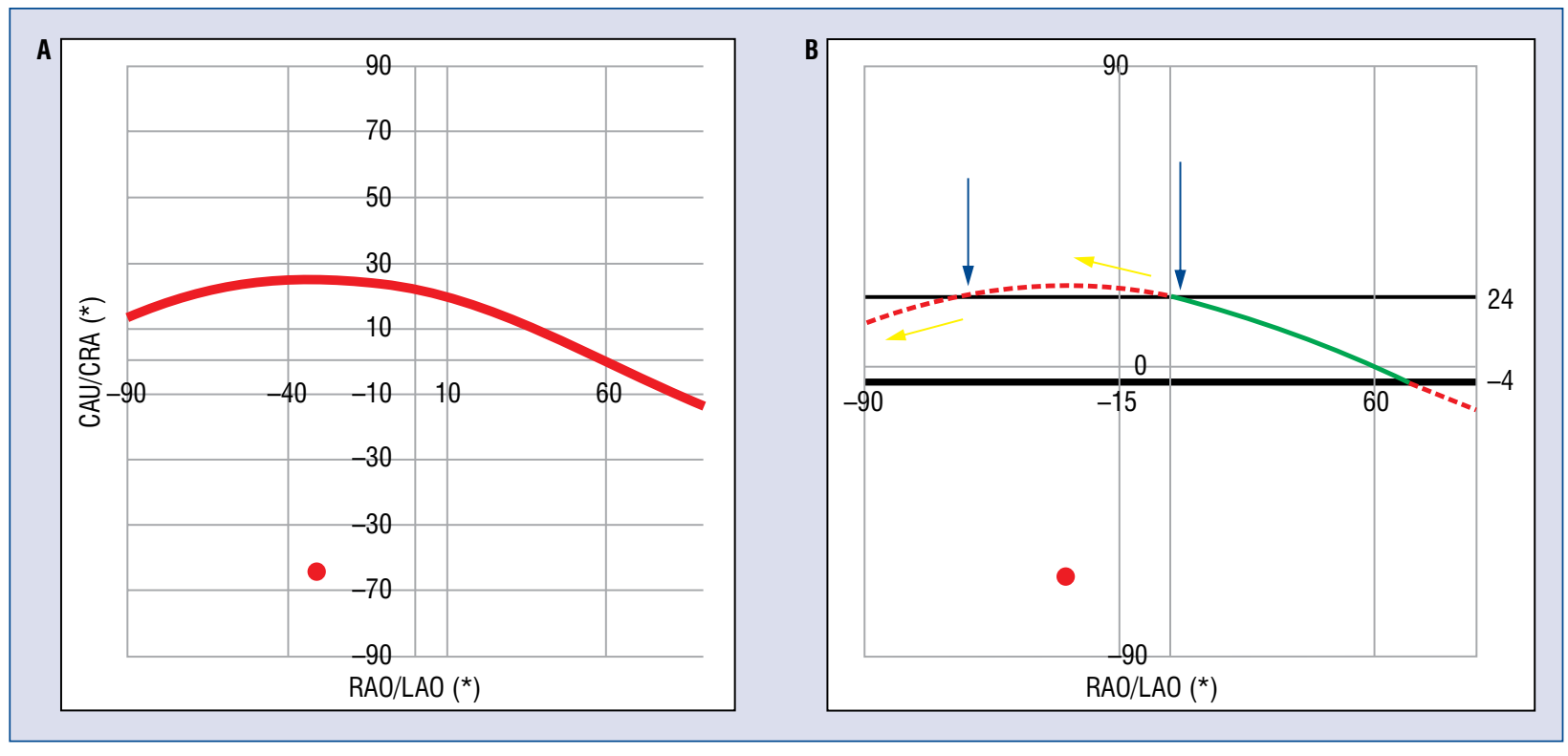

Figure 2. The optimal projection curve based on the determined en face angle; A. The optimal projection curve (red line) corresponding to the determined en face angle of the coronary ostium (red dot); $\mathbf{B}$. The range of the optimal projection curve in which the coronary ostium does not overlap with the adjacent aorta. Because determining this range is based on the caudal/cranial (CAU/CRA) angulation, it is crucial to notice that two different points on the S-curve can have the same CAU/CRA angulation value (blue arrows). They can be identified by their relation to the maximal angulation of the curve (yellow arrows). Red dotted lines — "obstructed" view, green line — "unobstructed" view.

The implanted stent of choice in non-ostial lesions is usually long enough to secure a reasonably safe margin on both sides of the lesion and minimize the risk of a geographic miss. In the case of ostial stenoses, one of the margins is completely absent. A deliberate decision to protrude the stent into the aorta may result in many longterm serious consequences. First, the protruding stent makes all subsequent interventions on that vessel much more difficult. It is harder to engage the vessel with the guiding catheter [20], and attempts to do so can deform the protruding struts, potentially increasing thrombotic risk. A recent analysis from the EXCEL trial clearly showed that PCI of a target vessel after left main (LM) stenting was a strong predictor of overall and cardiovascular mortality at 3 years [21]. Secondly, stent protrusion into the aorta could be a serious problem in patients who require surgical intervention in the future. The inability to selectively cannulate coronary vessels prevents antegrade cardioprotection. Stent struts can also make aortic valve replacement surgery extremely difficult. Intraoperative stent trimming poses the risk of deformation of the intracoronary part of the stent [6]. Finally, there is a continuously growing population of patients admitted for transcatheter aortic valve replacement (TAVR) procedures. In these patients, the protruding stent can be crushed by the delivery balloon or by the valve itself. That risk is best illustrated in Figure $6 \mathrm{C}, \mathrm{D}$, where the stent protruding from the LM was crushed by the bulky, calcified valve leaflet during transapical TAVR. The only available arterial access (right radial) was used for pig-tail catheter insertion, preventing LM protection with safety wire and coronary balloon. Despite the patent LIMA-LAD and Ao-Diagonal grafts, the complications turned out to be lethal.

On the other hand, in cases when the operator tries to place the stent precisely, without any protrusion, unintended incomplete coverage of the ostium is common, resulting in a greatly enhanced risk of subsequent restenosis (Fig. 6A, B). In one short series of patients after LM stenting, control MSCT revealed that the stent had been implanted optimally in relation to the ostium only in 3 out of 23 cases [5]. The precise positioning of stents in the coronary ostia is of paramount importance because it can substantially affect long-term clinical success. Unfortunately, angiographic views are plagued with parallax error, and finding a projection orthogonal to the individual aorto-ostial plane is very challenging. 


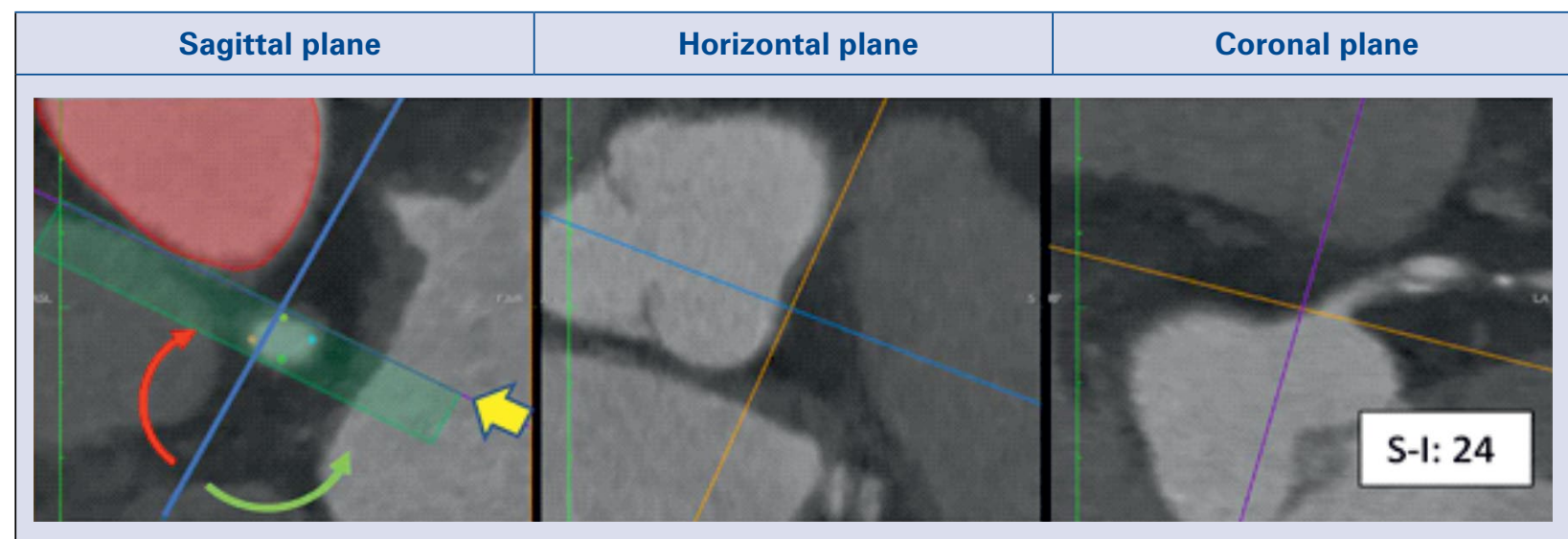

Sagittal view from Figure 1, step 6 - the left image - represents the en face plane of the coronary ostium. The $\mathrm{X}$-ray source is marked with the yellow arrow. The coronal plane window presents a "slice" of the projected angiographic view. Rotating the horizontal and frontal planes in the sagittal plane window, adjust the axes to the angle where further clockwise rotation (red arrow) would cause the silhouettes of the ostium (green rectangle) and the adjacent aorta (red area) to overlap. The superior-inferior (S-I) angle from the coronal plane window shows the real caudal/cranial (CAU/CRA) angulation of this borderline angiographic projection. Note: During rotation of the horizontal and frontal plane, the S-I angulation shown in the coronal plane window can indicate the same number twice (refer to Figure 2B, blue arrows). It is important to relate this point to the peak S-I angulation of the S-curve. Observing whether further clockwise rotation and overlapping the silhouettes of the ostium and the aorta would cause the S-I value to increase or to decrease (refer to Figure 2B, yellow arrows) enables identification of the correct point on the S-curve.

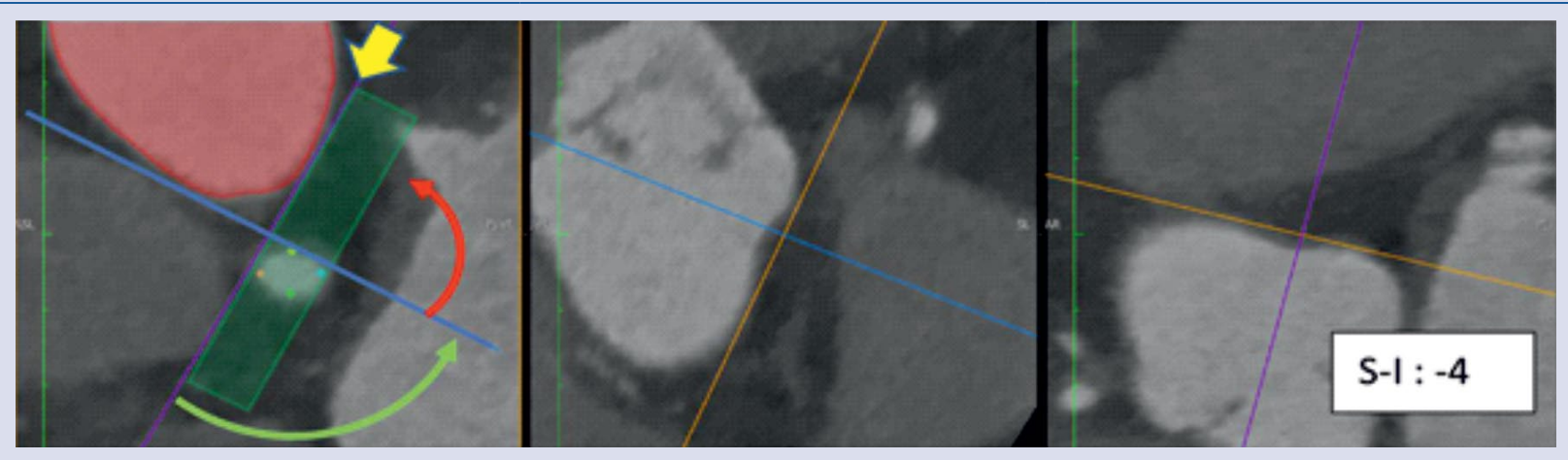

In the sagittal view window, rotate the horizontal and coronal planes counterclockwise to the angle where further rotation (red arrow) would cause the silhouettes of the ostium (green rectangle) and the adjacent aorta (red area) to overlap. The S-I angle from the coronal plane view shows the real CAU/CRA angulation of this borderline angiographic projection. The range between those two S-I angulations from the coronal plane view window corresponds to the range of the CAU/CRA angle of the optimal projection curve in which the coronary ostium does not overlap with the adjacent aorta (Fig. 2B).

Figure 3. Finding the range of S-curve in which the ostium and the aorta do not overlap with each other.

Table 1. Mean and $95 \%$ confidence intervals of en face angles of the coronary ostia, and interobserver difference.

\begin{tabular}{|c|c|c|c|c|c|}
\hline En face view & Overall & Observer 1 & Observer 2 & $\begin{array}{l}\text { Difference } \\
\text { between } \\
\text { observers }\end{array}$ & $\begin{array}{c}\text { Average range } \\
\text { of "unobstructed" } \\
\text { view }\end{array}$ \\
\hline LCA ostium & $\begin{array}{l}\text { RAO 29; CAU } 50 \\
\text { (RAO 24 - RAO 34; } \\
\text { CAU } 42 \text { - CAU 58) }\end{array}$ & $\begin{array}{l}\text { RAO 28; CAU } 49 \\
\text { (RAO 23 - RAO 33; } \\
\text { CAU } 41 \text { - CAU 57) }\end{array}$ & $\begin{array}{l}\text { RAO 29; CAU } 50 \\
\text { (RAO 24 - RAO 34; } \\
\text { CAU } 42 \text { - CAU 58) }\end{array}$ & $5(4-6)$ & RAO 0 - LAO 68 \\
\hline RCA ostium & $\begin{array}{l}\text { RAO 18; CRA } 5 \\
\text { (RAO } 13 \text { - RAO 23; } \\
\text { CAU } 2 \text { - CRA 12) }\end{array}$ & $\begin{array}{c}\text { RAO 19; CRA } 5 \\
\text { (RAO } 14 \text { - RAO 23; } \\
\text { CAU } 1 \text { - CRA 12) }\end{array}$ & $\begin{array}{l}\text { RAO 18; CRA } 5 \\
\text { (RAO } 12 \text { - RAO 24; } \\
\text { CAU } 2 \text { - CRA 12) }\end{array}$ & $7(6-8)$ & $\begin{array}{c}\text { RAO } 90 \text { - RAO } 37 \\
\text { and } \\
\text { LAO } 48 \text { - LAO } 90\end{array}$ \\
\hline
\end{tabular}

$\mathrm{CAU}$ - caudal angulation; CRA — cranial angulation; LAO — left anterior oblique angulation; RAO — right anterior oblique angulation 


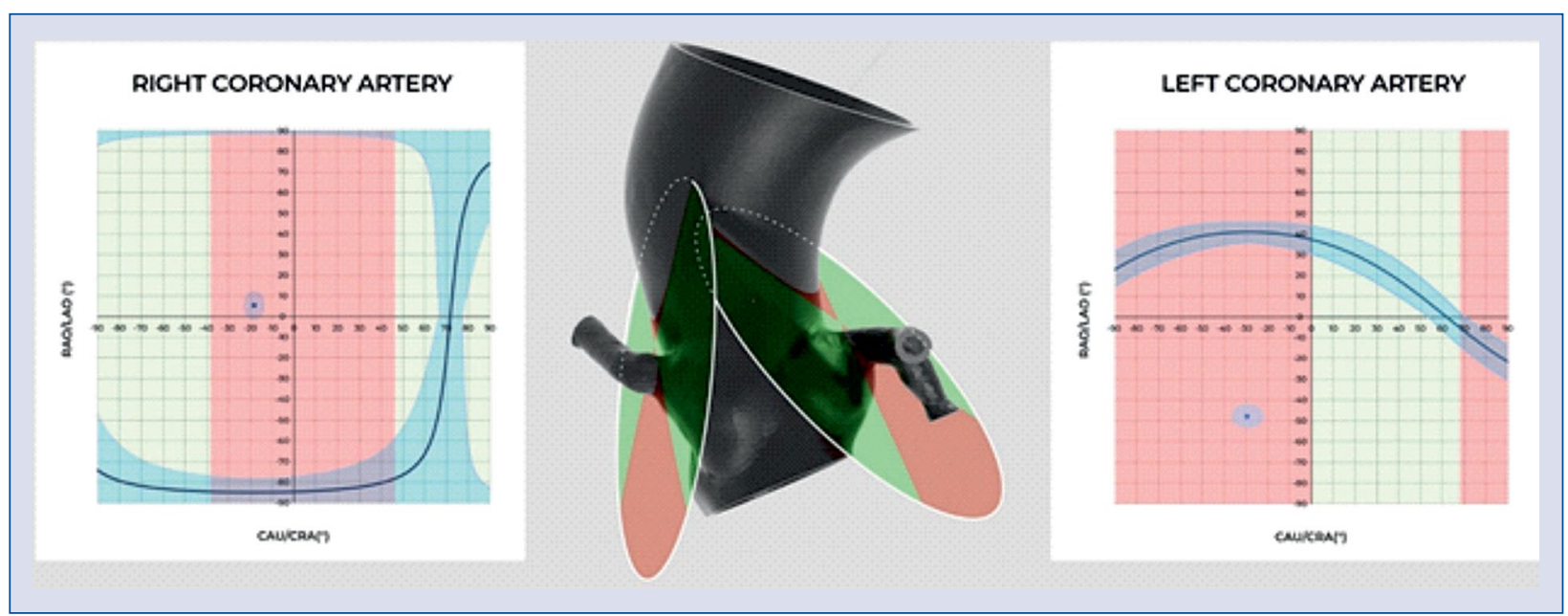

Figure 4. En face angles of the right and left coronary ostia and their corresponding optimal projection curves (mean and $95 \%$ confidence interval). En face angle — blue dot. Optimal projection curve - blue line. The red area indicates the range in which the coronary ostium and the aortic root overlap. Central panel represents schematic illustration of coronary ostia plane in relation to the aortic root.

Accurate image acquisition by using MSCT facilitates PCIs of ostial lesions

Multislice computed tomography provides complete information about the spatial relations between the aortic root, ascending aorta, and coronary vasculature. MSCT data are successfully used for device sizing, procedure planning, and angiographic view optimization during structural cardiac procedures. However, their utilization to facilitate coronary interventions has hitherto been limited, although promising [15, 22]. There are no publications defining an accurate angiographic view for a subsequent coronary procedure based on MSCT image acquisition.

In our study, we propose a simple way of establishing the en face angle of the aorto-ostial plane and the corresponding optimal projection curve using a standard DICOM viewer. Results from a group of 30 patients show that the angle of the coronary aorto-ostial plane and its relation to the adjacent aorta are highly variable and patient-specific. In some patients, a large portion of the optimal projection curve of the coronary ostium overlaps with the adjacent aorta, so the range of the "clear" viewing angle is quite limited. In such cases, the ostium is located in the concave part of the aortic root complex (Fig. 7A, C). In other patients in whom the coronary artery has its origin at the convex part of the sinus of Valsalva, it does not overlap with the aorta at any point (Fig. 7B, C). In these scenarios, one should opt for the projection with the smallest CRA/CAU angle.
Taking into account the high variability of coronary ostial planes and aortic configurations, an individual S-curve of the treated ostium should be calculated if an MSCT examination is available. Sometimes in the case of a funnel-shaped ostium, it may be difficult to precisely define anatomical borders. The specific ostium definition can then be fine-tuned according to the planned stenting strategy, i.e. orthogonal to the ostium at the level of the desired stent edge position.

Hitherto, the data regarding the spatial and anatomical location of the coronary ostia in patients admitted for PCI were often not available. However, since MSCT is now a first-line tool for diagnosing $\mathrm{CAD}$ in patients with chronic coronary syndromes [23], the availability of these data will be expanding as well. MSCT image interpretation should, therefore, be another skill in the interventional cardiologist's pocket. Nowadays, PCI operators should not just rely on the radiologist's report but should also be able to review MSCT exams by themselves, in order to assess anatomical subtleties of every individual patient and optimize the treatment strategy and viewing angles.

However, in most patients admitted for PCI of the LCA or RCA ostium, procedures are performed routinely only based on the clinical experience and judgment of the operator. This also applies to the choice of angiographic projection. The mean en face angles and corresponding optimal projection curves from our study roughly reflect current clinical practice. The optimal projection for stenting the 


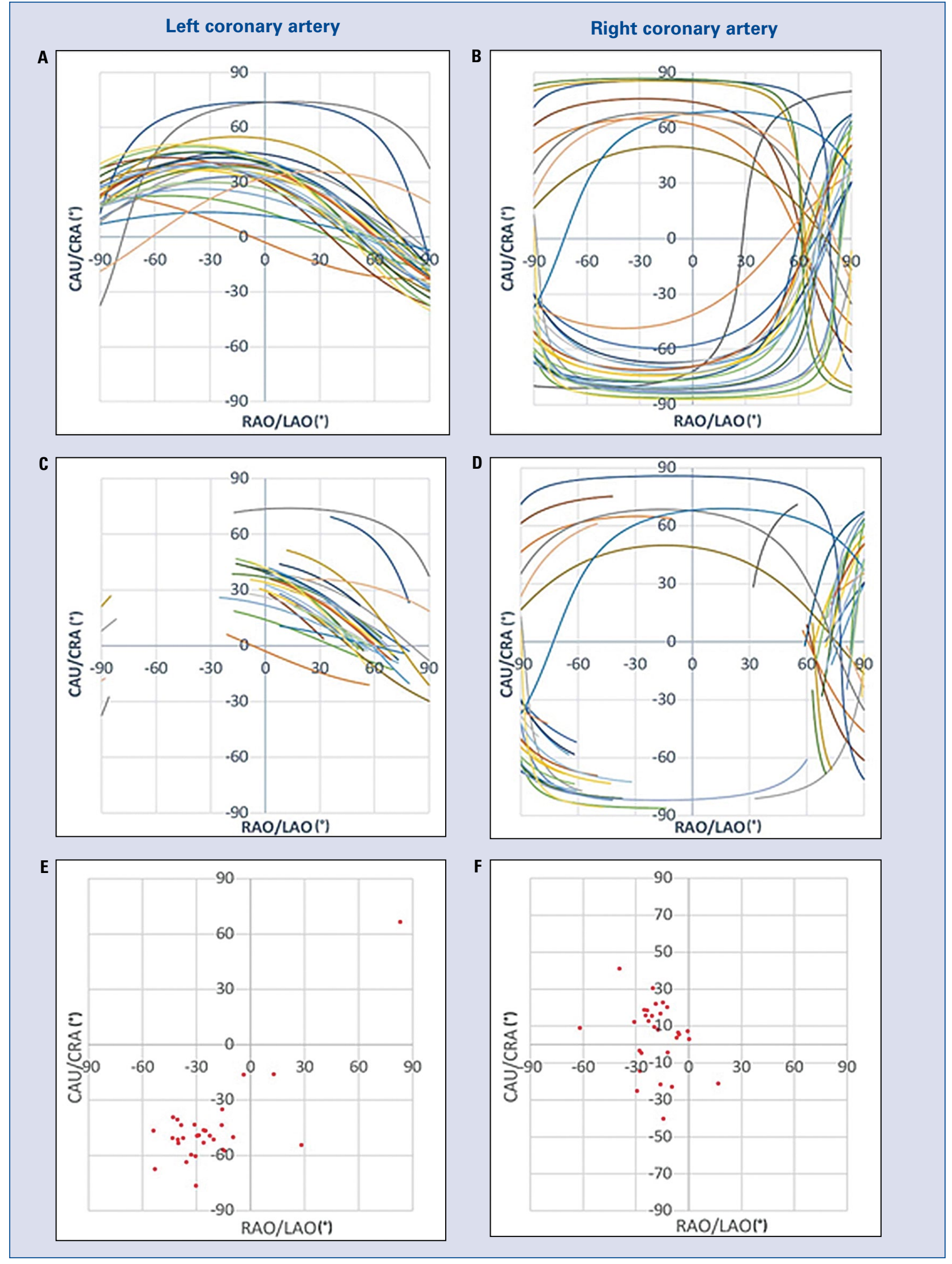

Figure 5. Individual optimal projection curves and en face angles in the study cohort. Optimal projection curves for the ostia of the left (A) and right coronary arteries (B). The range of the optimal projection curves in which neither the left (C) nor right coronary ostium (D) overlaps with the adjacent aorta. En face angles of left (E) and right coronary ostia (F). 


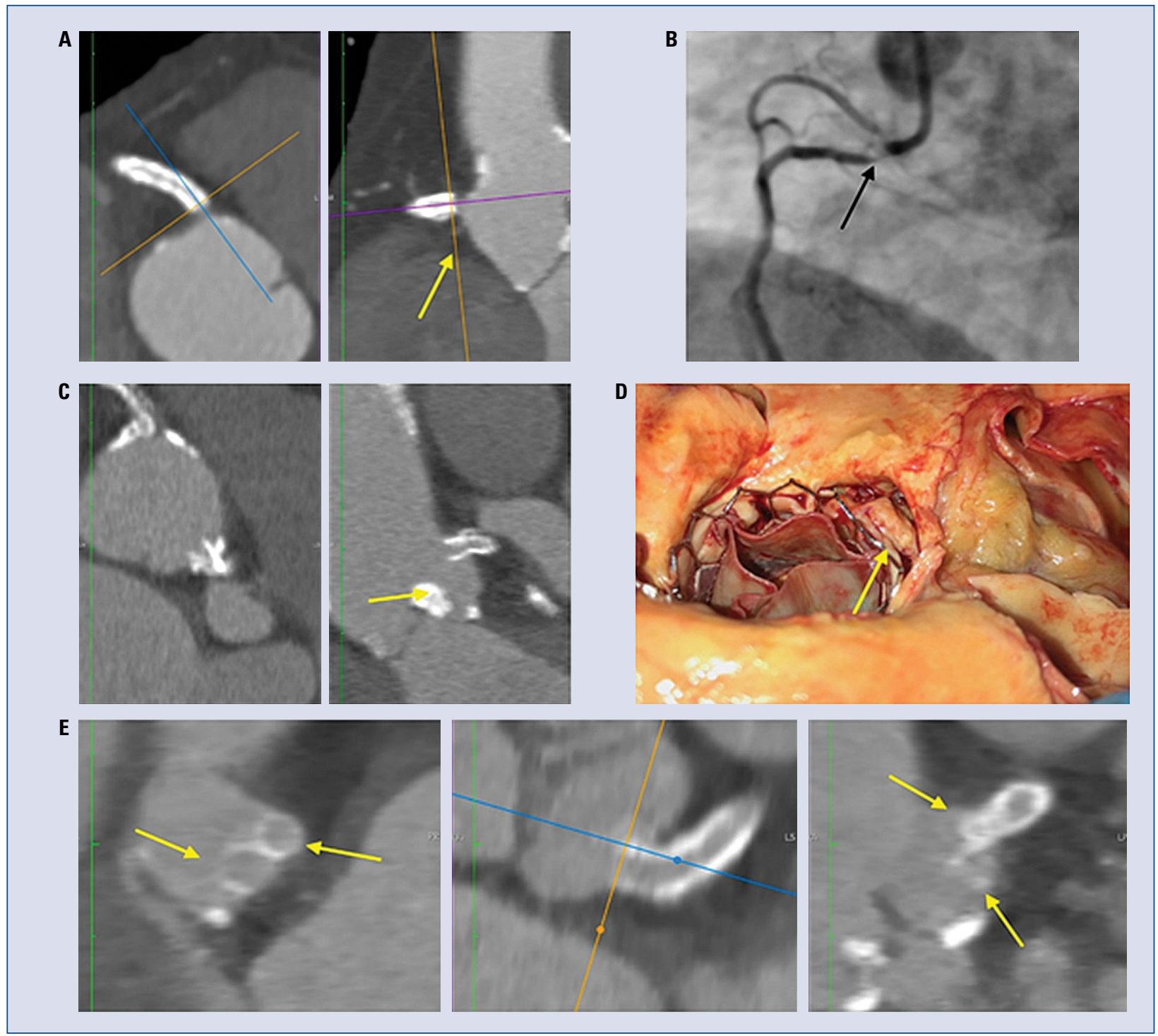

Figure 6. Clinical examples of the suboptimal stent placement in the aorto-coronary ostial lesions. Geographic miss (arrow), stent in the right coronary artery (RCA) implanted too deeply (A); subsequent restenosis in the RCA (arrow) (B); stent protruding from left main into the aorta (arrow) (C); protruding stent crushed (arrow) by the calcified, widened aortic leaflet on postmortem examination (D); two stents protruding from the left coronary artery to the aorta (arrows) accidental finding in the patient admitted for transcatheter aortic valve replacement $(\mathbf{E})$.

LM ostium is usually in the LAO projection with cranial angulation. The best strategy for finding this optimal projection is to start at around LAO $30-40^{\circ}$, CRA $30^{\circ}$ and proceed caudally. In the case of the right coronary ostium plane, most optimal projections will be between $\mathrm{LAO} 60^{\circ}$ and $80^{\circ}$ with slight cranial angulation. The best strategy seems to be to start in LAO $60^{\circ}, \mathrm{CRA} 10^{\circ}$ and then move east toward LAO $90^{\circ}$.

Although two-dimensional (2D) angiographic images are completely spatially unoriented compared to MSCT data, there is a "life hack" solution for finding a projection perpendicular to the coronary ostium plane. If one aligns the tip of the guiding catheter with the coronary ostium and then finds the projection in which the catheter looks like a straight line with its tip circular, then this corresponds roughly to the ostial plane en face angle.

No matter what the CRA/CAU angulation of that ostial plane en face angle is, the perpendicular projection will always be located at the position of $\mathrm{CRA} / \mathrm{CAU} 0^{\circ}$, with $\mathrm{RAO} / \mathrm{LAO}$ angulation equal to the en face plane RAO/LAO value plus or minus 90 . That may be the starting point for finding the optimal projection and/or calculating the expected/ /approximated S-curve. However, one should keep 


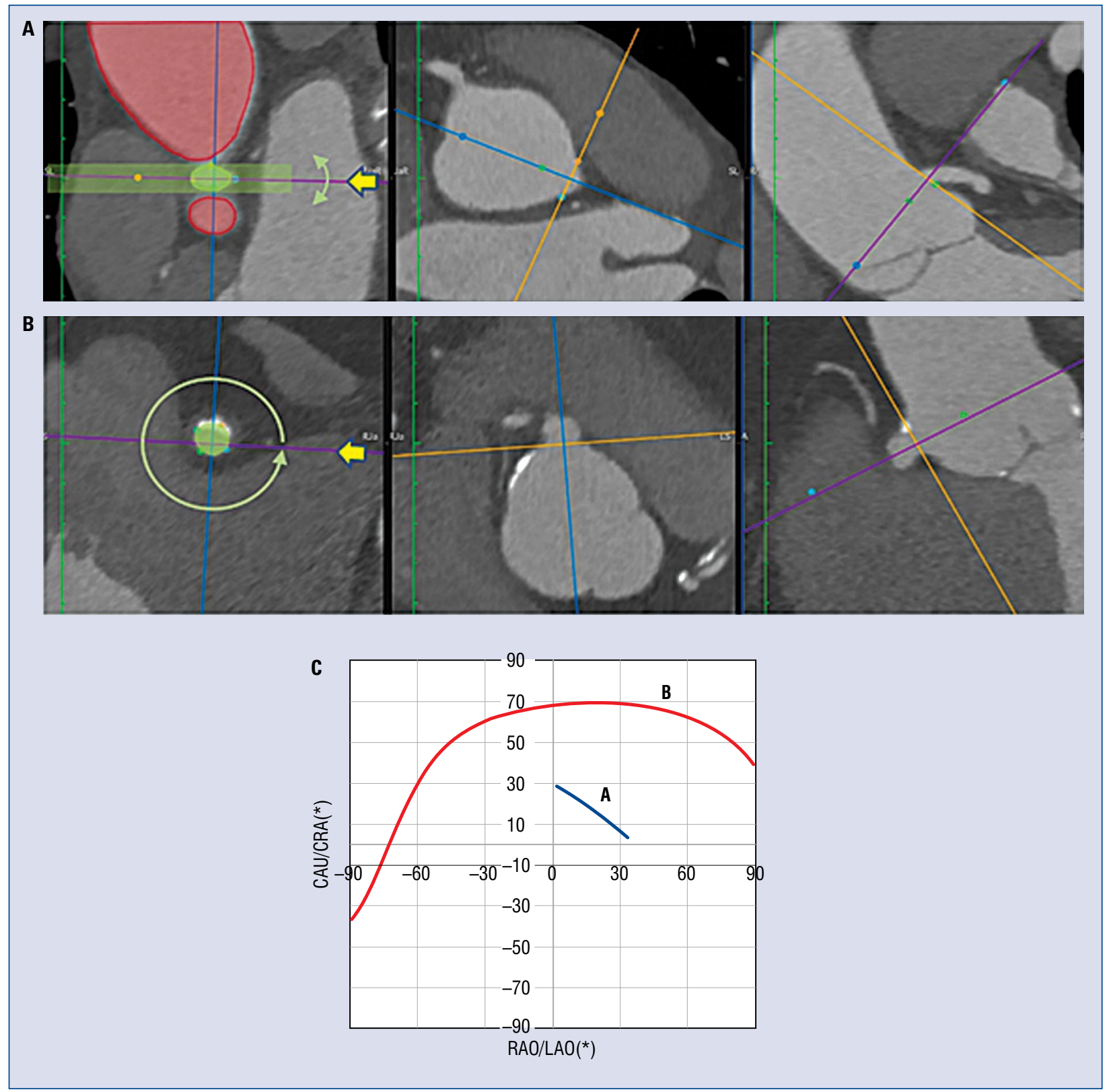

Figure 7. Examples of different aortic root configurations and their impact on the S-curve. Two examples of different aortic root configurations; A. A narrow "angiographic window" of the left coronary artery (LCA) ostium (green oval) with a very limited range of possible angulation (green arrow) of the X-ray source (yellow arrow) (adjacent aorta — red area); B. A wide "angiographic window" of the right coronary artery (RCA) ostium (green circle), offering a 360-degree view; C. The range of the corresponding S-curves in which the coronary ostium does not overlap with the adjacent aorta. In the case of the LCA (blue curve), angiographic viewing angles are limited, but are paradoxically "standard" and easy to achieve, while in the case of the RCA ostium (orange curve), its S-curve is quite atypical and, despite the full range, the degree of cranial angulation in standard left anterior oblique projections makes them unfeasible.

in mind that the tip of the catheter engaged in the coronary ostium is usually oriented upwards. This tendency can be corrected by pushing on the coronary guidewire for better alignment.

\section{Limitations of the study}

The main limitation of our study is that the study population consisted of a small group of patients with suspected coronary disease, admitted 
for diagnostic CTA, who did not have aorto-ostial lesions, mandating intervention. Thus, the presented data remain experimental. In addition, the data has not been prospectively validated. Third, the method itself is cumbersome. Dedicated software would greatly facilitate the determination of the optimal angle of the coronary ostia. Finally, in the case of structural interventions, a predefined "implantation" projection can easily be corrected during the procedure. This may not be the case with the coronary ostial plane, due to its small size. Altogether, the clinical significance of our method remains to be established.

\section{Conclusions}

To overcome the technical challenges of aorto-ostial PCIs, we propose the first systematic step-by-step approach to determine individual fluoroscopic viewing angles for precise stenting by using MSCT image acquisition. The present study shows that, given the substantial variability of the aortic root anatomy, patient-specific implantation angles determined before intervention may essentially facilitate the procedure. The question of whether we should perform MSCT before aorto-ostial PCI for the sake of procedure planning and optimizing its results remains open and needs further prospective investigations.

\section{Conflict of interest: None declared}

\section{References}

1. Mavromatis K, Ghazzal Z, Veledar E, et al. Comparison of outcomes of percutaneous coronary intervention of ostial versus nonostial narrowing of the major epicardial coronary arteries. Am J Cardiol. 2004; 94(5): 583-587, doi: 10.1016/j.amjcard.2004.05.020, indexed in Pubmed: 15342287.

2. Freeman M, Clark DJ, Andrianopoulos N, et al. Outcomes after percutaneous coronary intervention of ostial lesions in the era of drug-eluting stents. Catheter Cardiovasc Interv. 2009; 73(6): 763-768, doi: 10.1002/ccd.21941, indexed in Pubmed: 19309731.

3. Jokhi P, Curzen N. Percutaneous coronary intervention of ostial lesions. EuroIntervention. 2009; 5(4): 511-514, doi: 10.4244/ eijv5i4a81, indexed in Pubmed: 19755342.

4. Vaduganathan M, Harrington RA, Stone GW, et al. Cangrelor with and without glycoprotein iib/iiia inhibitors in patients undergoing percutaneous coronary intervention. J Am Coll Cardiol. 2017; 69(2): 176-185, doi: 10.1016/j.jacc.2016.10.055, indexed in Pubmed: 28081827.

5. Rubinshtein R, Ben-Dov N, Halon DA, et al. Geographic miss with aorto-ostial coronary stent implantation: insights from highresolution coronary computed tomography angiography. EuroIntervention. 2015; 11(3): 301-307, doi: 10.4244/EIJV11I3A57, indexed in Pubmed: 24694540.

6. Nakamura M, Minakata K, Yamazaki K, et al. Aortic valve replacement in patients with protruding coronary artery stents. J Cardiol Cases. 2014; 10(6): 213-215, doi: 10.1016/j.jccase.2014.07.013, indexed in Pubmed: 30534246.

7. Wintzer-Wehekind J, Larose E, Rodés-Cabau J, et al. Coronary Stent Protruding in the Ascending Aorta: Multimodality Imaging
Evaluation. JACC Cardiovasc Interv. 2020; 13(2): 263-264, doi: 10.1016/j.jcin.2019.07.052, indexed in Pubmed: 31563684.

8. Achenbach S. Cardiac CT: state of the art for the detection of coronary arterial stenosis. J Cardiovasc Comput Tomogr. 2007; 1(1): 3-20, doi: 10.1016/j.jcct.2007.04.007, indexed in Pubmed: 19083871.

9. Saremi F, Achenbach S. Coronary plaque characterization using CT. AJR Am J Roentgenol. 2015; 204(3): W249-W260, doi: 10.2214/AJR.14.13760, indexed in Pubmed: 25714309.

10. Hoffmann U, Truong Q, Schoenfeld D, et al. Coronary CT Angiography versus Standard Evaluation in Acute Chest Pain. N Engl J Med. 2012; 367(4): 299-308, doi: 10.1056/nejmoa1201161.

11. Blanke P, Weir-McCall JR, Achenbach S, et al. Computed tomography imaging in the context of transcatheter aortic valve implantation (tavi)/transcatheter aortic valve replacement (TAVR): an expert consensus document of the society of cardiovascular computed tomography. JACC Cardiovasc Imaging. 2019; 12(1): 1-24, doi: 10.1016/j.jcmg.2018.12.003, indexed in Pubmed: 30621986.

12. Thériault-Lauzier P, Andalib A, Martucci G, et al. Fluoroscopic anatomy of left-sided heart structures for transcatheter interventions: insight from multislice computed tomography. JACC Cardiovasc Interv. 2014; 7(9): 947-957, doi: 10.1016/j. jcin.2014.06.002, indexed in Pubmed: 25129665.

13. Pighi M, Thériault-Lauzier P, Alosaimi H, et al. Fluoroscopic Anatomy of Right-Sided Heart Structures for Transcatheter Interventions. JACC Cardiovasc Interv. 2018; 11(16): 1614-1625, doi: 10.1016/j.jcin.2018.03.050, indexed in Pubmed: 30139469.

14. Thériault-Lauzier P, Spaziano M, Vaquerizo B, et al. Computed Tomography for Structural Heart Disease and Interventions. Interv Cardiol. 2015; 10(3): 149-154, doi: 10.15420/ ICR.2015.10.03.149, indexed in Pubmed: 29588693.

15. Pesenti-Rossi D, Chouli M, Gharbi M, et al. Coronary aortoostial stenosis analysed by multislice computed tomography: a new tool for percutaneous coronary intervention? EuroIntervention. 2011; 6(6): 717-721, doi: 10.4244/EIJV6I6A121, indexed in Pubmed: 21205594.

16. Hammas S, Amato A, Amabile N, et al. Use of multislice computed tomography angiography in percutaneous coronary intervention. Int Cardiol. 2011; 3(4): 483-492, doi: 10.2217/ ica.11.55.

17. Xiong TY, Pighi M, Thériault-Lauzier P, et al. Optimal fluoroscopic viewing angles of right-sided heart structures in patients with tricuspid regurgitation based on multislice computed tomography. EuroIntervention. 2019; 15(10), doi: 10.4244/EIJD-19-00618, indexed in Pubmed: 31746757.

18. Zampieri P, Colombo A, Almagor Y, et al. Results of coronary stenting of ostial lesions. Am J Cardiol. 1994; 73(12): 901-903, doi: 10.1016/0002-9149(94)90822-2, indexed in Pubmed: 8184820 .

19. Rathore S, Terashima M, Katoh O, et al. Predictors of angiographic restenosis after drug eluting stents in the coronary arteries: contemporary practice in real world patients. EuroIntervention. 2009; 5(3): 349-354, doi: 10.4244/v5i3a55, indexed in Pubmed: 19736160.

20. Uehara Y, Shimizu M, Yoshimura M. A novel technique for catheter engagement of protruding aorto-ostial stent. Catheter Cardiovasc Interv. 2014; 83(7): 1093-1096, doi: 10.1002/ccd.25274, indexed in Pubmed: 24174303.

21. Giustino G, Serruys P, Sabik J, et al. Mortality after repeat revascularization following PCI or CABG for left main disease. JACC: Cardiovascular Interventions. 2020; 13(3): 375-387, doi: 10.1016/j.jcin.2019.09.019.

22. Jones DA, Castle EV, Beirne AM, et al. Computed tomography cardiac angiography for planning invasive angiographic procedures in patients with previous coronary artery bypass grafting. EuroIntervention. 2020; 15(15): e1351-e1357, doi: 10.4244/EIJ-D-18-01185, indexed in Pubmed: 31235457.

23. Knuuti J, Wijns W, Achenbach S, et al. 2019 ESC guidelines for the diagnosis and management of chronic coronary syndromes. Eur Heart J. 2020; 41(3): 407-477, doi: 10.1093/eurheartj/ ehz425, indexed in Pubmed: 31504439. 